\title{
ON SPECIAL CONCIRCULAR $R$-LIE-RECURRENCE IN SPECIAL FINSLER SPACES
}

\author{
G.P. Yadav ${ }^{1}$, P.N. Pandey ${ }^{2}$ \\ ${ }^{1,2}$ Department of Mathematics \\ University of Allahabad \\ Allahabad, 211002, INDIA
}

\begin{abstract}
In this paper we discuss a special concircular $R$-Lie-recurrence in special Finsler spaces such as $R$-recurrent, $R$-symmetric, $R$-birecurrent and $R$-bisymmetric. Apart from other theorems, it is being proved that an $R$-recurrent Finsler space can not admit a special concircular $R$-Lie-recurrence while a non-flat $R$-symmetric Finsler space $F_{n}(n>2)$ admitting a special concircular $R$-Lie-recurrence is necessarily of constant Riemannian curvature.
\end{abstract}

AMS Subject Classification: 53B40.

Key Words: Finsler space, $R$-Lie-recurrence, Riemannian curvature, special concircular vector field, R-recurrent Finsler space.

\section{Introduction}

In 1982, P. N. pandey [5] introduced the concept of Lie-recurrence in a Finsler Space. In 1992, K. L. Duggal [3] studied the Lie-recurrence in a Riemannian space with its application to fluid space time but he used the term curvature inheriting symmetry in place of Lie-recurrence. He also used the theory to the study of fluid space time. Since then both the terms (Lie-recurrence and curvature inheriting symmetry) are in use (see [1] and [11]-[14]). P. N. Pandey and vaishali pandey [12] discussed a $K$-curvature inheritance, $K$-projective Lierecurrence and special concircular $K$-Lie-recurrence in a Finsler space.

Received: $\quad$ October 13, 2016

Revised: $\quad$ December 21, 2016

Published: $\quad$ March 28, 2017

$\S$ Correspondence author (c) 2017 Academic Publications, Ltd. url: www.acadpubl.eu 
The authors (P. N. pandey and Vaishali Pandey) [13] studied a K-Lierecurrence in a Finsler space. C. K. Mishra and Gautam Lodhi [1] discussed curvature inheriting symmetry and Ricci-inheriting symmetry in a Finsler space and obtained some results. In this paper we have discussed a special concircular $R$-Lie-recurrence in special Finsler spaces such as $R$-recurrent, $R$-symmetric, $R$ birecurrent and $R$-bisymmetric.

\section{Preliminaries}

Let $F_{n}$ be an $n$-dimensional Finsler space equipped with a metric function $F$ satisfying the requisite conditions [2]. The relation between the metric tensor $g_{i j}$ of the Finsler space $F_{n}$ and the metric function $F$ are given by

$$
\text { (a) } g_{i j}=\frac{1}{2} \dot{\partial}_{i} \dot{\partial}_{j} F^{2}, \text { (b) } g_{i j} y^{i} y^{j}=F^{2}
$$

where $\dot{\partial}_{i} \equiv \frac{\partial}{\partial y^{2}}$.

The Cartan $h$-covariant derivative of an arbitrary vector field $T^{i}$ with respect to connection coefficients $\Gamma_{j k}^{*}$ is given by

$$
T_{\mid k}^{i}=\partial_{k} T^{i}-\left(\dot{\partial}_{r} T^{i}\right) \Gamma_{h k}^{* r} y^{h}+T^{r} \Gamma_{r k}^{* i},
$$

where $\partial_{k} \equiv \frac{\partial}{\partial x^{k}}$.

The Cartan $v$-covariant derivative of an arbitrary vector field $T^{i}$ is given by

$$
\left.T^{i}\right|_{k}=\dot{\partial}_{k} T^{i}+T^{r} C_{r k}^{i},
$$

where $C_{r k}^{i}=g^{i j} C_{j r k}$. The tensor $C_{j r k}$ is called Cartan tensor and defined as $C_{j r k}=\frac{1}{2} \dot{\partial}_{j} g_{r k}$.

The Ricci commutation formula for $h$-covariant derivative is given by

$$
T_{|h| k}^{i}-T_{|k| h}^{i}=T^{r} R_{r h k}^{i}-\left.T^{i}\right|_{j} R_{s h k}^{j} y^{s},
$$

where $R_{r h k}^{i}=\partial_{k} \Gamma_{r h}^{*}-\left(\dot{\partial}_{t} \Gamma_{r h}^{* i}\right) \Gamma_{s k}^{*} \dot{x}^{s}+\Gamma_{r h}^{*} \Gamma_{t k}^{* i}+C_{r m}^{i}\left(\partial_{k} G_{h}^{m}+G_{h}^{t} G_{t k}^{m}\right)$ $-\partial_{h} \Gamma_{r k}^{*}+\left(\dot{\partial}_{t} \Gamma_{r k}^{* i}\right) \Gamma_{s h}^{* t} \dot{x}^{s}-\Gamma_{r k}^{*} \Gamma_{t h}^{* i}-C_{r m}^{i}\left(\partial_{h} G_{k}^{m}+G_{k}^{t} G_{t h}^{m}\right)$.

The tensor $R_{j k h}^{i}$ is called $h$-curvature tensor. This tensor is skew-symmetric in last two lower indices and positively homogeneous of degree zero in $y^{i}$. Cartan curvature tensor $K_{j k h}^{i}$, Cartan $h$-curvature tensor $R_{j k h}^{i}$ are related by

$$
R_{j k h}^{i}=K_{j k h}^{i}+C_{j m}^{i} H_{k h}^{m},
$$


where $H_{k h}^{m}=\partial_{h} G_{k}^{m}-\partial_{k} G_{h}^{m}+G_{k}^{r} G_{r h}^{m}-G_{h}^{r} G_{r k}^{m}$.

Transvecting (5) by $y^{j}$ and using the fact $C_{j m}^{i} y^{j}=0$, we get

$$
R_{j k h}^{i} y^{j}=H_{k h}^{i} .
$$

The tensor $H_{k h}^{i}$ is connected with Berwald deviation tensor $H_{h}^{i}$ by

$$
\text { (a) } y^{k} H_{k h}^{i}=H_{h}^{i} \text {, (b) } \dot{\partial}_{k} H_{h}^{i}-\dot{\partial}_{h} H_{k}^{i}=3 H_{k h}^{i} \text {. }
$$

Berwald deviation tensor satisfies the following:

$$
\text { (a) } g_{i k} H_{h}^{i}=g_{i h} H_{k}^{i} \text {, (b) } y_{i} H_{h}^{i}=0 \text {, (c) } H_{i}^{i}=(n-1) H,
$$

where $y_{i}=g_{i j} y^{j}$ and $H$ is scalar curvature.

The commutation formula for the operators of partial differentiation with respect to $y^{k}$ and $h$-covariant differentiation is given by

$$
\dot{\partial}_{k}\left(T_{\mid h}^{i}\right)-\left(\dot{\partial}_{k} T^{i}\right)_{\mid h}=T^{r} \dot{\partial}_{k} \Gamma_{r h}^{* i}-\left(\dot{\partial}_{r} T^{i}\right)\left(\dot{\partial}_{k} \Gamma_{s h}^{* r}\right) y^{s} .
$$

Let us consider an infinitesimal transformation

$$
\bar{x}^{i}=x^{i}+\epsilon v^{i}\left(x^{j}\right),
$$

generated by a contravariant vector field $v^{i}\left(x^{j}\right)$ which depends on position coordinates only. $\epsilon$ appearing in (10) is an infinitesimal constant.

The Lie-derivative of an arbitrary tensor $T_{j}^{i}$ with respect to the infinitesimal transformation (10) is given by [4]

$$
£ T_{j}^{i}=T_{j \mid r}^{i} v^{r}-T_{j}^{r} v_{\mid r}^{i}+T_{r}^{i} V_{\mid j}^{i}+\left(\dot{\partial}_{r} T_{j}^{i}\right) v_{\mid s}^{r} y^{s} .
$$

The commutation formula for the operators $£$ and $\dot{\partial_{h}}$ is given by

$$
\dot{\partial_{h}} £ \Omega-£ \dot{\partial_{h}} \Omega=0,
$$

where $\Omega$ is any geometrical object. An infinitesimal transformation (10) is Lierecurrence or $H$-Lie-recurrence if the Lie-derivative of Berwald curvature tensor $H_{j k h}^{i}$ of the Finsler space satisfies

$$
£ H_{j k h}^{i}=\phi H_{j k h}^{i},
$$

where $\phi$ is a non-zero scalar field [5]. In view of this concept, the infinitesimal transformation (10) is called $R$-Lie-recurrence if the Lie-derivative of Cartan $h$-curvature tensor satisfies [13]

$$
£ R_{j k h}^{i}=\phi R_{j k h}^{i}, \quad \phi \neq 0 .
$$


A vector field $v^{i}$ in the Finsler space $F_{n}$ is said to be special concircular if

$$
\text { (a) } v_{\mid k}^{i}=\rho \delta_{k}^{i},\left.\quad(\mathrm{~b}) v^{i}\right|_{k}=0
$$

where $\rho=\rho(x)[13]$.

\section{Special Concircular R-Lie-Recurrence}

Theorem 1. An $R$-recurrent Finsler space $F_{n}(n>2)$ can not admit a special concircular $R$-Lie-recurrence.

Proof. Let us consider a Finsler space admitting the infinitesimal transformation (10) generated by a special concircular vector field $v^{i}\left(x^{j}\right)$. Differentiating $(15 a)$ covariantly with respect to $x^{h}$,we get

$$
v_{|k| h}^{i}=\rho_{h} \delta_{k}^{i}
$$

where $\rho_{h}=\rho_{\mid h}$. Taking skew-symmetric part of (16) and utilizing commutation formula (4) and using (15b), we have

$$
v^{r} R_{r k h}^{i}=\rho_{h} \delta_{k}^{i}-\rho_{k} \delta_{h}^{i}
$$

Contraction of indices $i$ and $h$ in (17) gives

$$
v^{r} R_{r k}=-(n-1) \rho_{k}
$$

where $R_{r k}$ is Ricci tensor defined as $R_{r k}=R_{r k h}^{h}$. From equations (17) and (18), we may write

$$
v^{r} R_{r k h}^{i}=\frac{1}{n-1} v^{r}\left(R_{r k} \delta_{h}^{i}-R_{r h} \delta_{k}^{i}\right)
$$

which implies

$$
\begin{gathered}
\left\{R_{r k h \mid m}^{i}-\frac{1}{n-1}\left(R_{r k \mid m} \delta_{h}^{i}-R_{r h \mid m} \delta_{k}^{i}\right)\right\} v^{r} \\
+\rho\left\{R_{m k h}^{i}-\frac{1}{n-1}\left(R_{m k} \delta_{h}^{i}-R_{m h} \delta_{k}^{i}\right)\right\}=0 .
\end{gathered}
$$

Let the Finsler space $F_{n}$ be $R$-recurrent characterized by

$$
R_{j k h \mid m}^{i}=\lambda_{m} R_{j k h}^{i},
$$


where $\lambda_{m}$ are components of a non-zero covariant vector field [7]. Contracting the indices $i$ and $h$ in (21), we have

$$
R_{j k \mid m}=\lambda_{m} R_{j k}
$$

From (19), (20), (21) and (22), we get

$$
R_{m k h}^{i}=\frac{1}{n-1}\left(R_{m k} \delta_{h}^{i}-R_{m h} \delta_{k}^{i}\right)
$$

Transvecting (23) by $y^{m}$ and using (6) and $R_{m k} y^{m}=H_{k}$, we have

$$
H_{k h}^{i}=\frac{1}{n-1}\left(H_{k} \delta_{h}^{i}-H_{h} \delta_{k}^{i}\right)
$$

Transvecting (24) by $y_{i}$ and using $y_{i} H_{k h}^{i}=0$ [8], we get $H_{k} y_{h}=H_{h} y_{k}$, which implies

$$
H_{k}=\frac{n-1}{F^{2}} H y_{k}
$$

for $H_{h} y^{h}=(n-1) H$ and $y_{h} y^{h}=F^{2}$. In view of (25), (24) may be rewritten as

$$
H_{k h}^{i}=R\left(y_{k} \delta_{h}^{i}-y_{h} \delta_{k}^{i}\right)
$$

where $R=\frac{H}{F^{2}}$. In view of Berwald theorem [2], equation (26) implies that $R$ is a constant and the space $F_{n}(n>2)$ is of constant Riemannian curvature. Differentiating (26) covariantly, we find

$$
H_{K h \mid m}^{i}=0
$$

for $y_{k \mid m}=0$.

Transvecting (21) by $y^{j}$ and using equation (6), we get $H_{k h \mid m}^{i}=\lambda_{m} H_{k h}^{i}$, which in view of (27), implies $\lambda_{m}=0$, a contradiction. Therefore, an $R$-recurrent Finsler space $F_{n}(n>2)$ can not admit a special concircular infinitesimal transformation.

Definition 2. A Finsler space $F_{n}(n>2)$ be $R$-symmetric characterized by $[6]$

$$
R_{j k h \mid m}^{i}=0
$$

Theorem 3. A special concircular $R$-Lie-recurrence in a non-flat $R$ symmetric Finsler space $F_{n}(n>2)$ is an $H$-Lie-recurrence and the $R$-symmetric Finsler space is necessarily of constant Riemannian curvature. 
Proof. Then, equation (20) implies equation (23). Adapting the above proceedure, we may show that equation (23) implies that the space $F_{n}(n>2)$ is of constant Riemannian curvature if it is non-flat. Suppose that the special concircular transformation (10) is a Lie-recurrence in the $R$-symmetric Finsler space $F_{n}(n>2)$. Then we have equation (14). In view of equation (11), equation (14) may be written as

$$
R_{j k h \mid r}^{i} v^{r}+\left(\dot{\partial}_{r} R_{j k h}^{i}\right) v_{\mid s}^{r} y^{s}-R_{j k h}^{r} v_{\mid r}^{i}+R_{r k h}^{i} v_{\mid j}^{r}+R_{j r h}^{i} v_{\mid k}^{r}+R_{j k r}^{i} v_{\mid h}^{r}=\phi R_{h j k}^{i} .
$$

Using equations (16), (28) and the fact that the curvature tensor $R_{h j k}^{i}$ is positively homogeneous of degree zero in $y^{j}$, we get $\phi=2 \rho$ if the space is non-flat. Since $\rho$ is independent of $y^{i}$ and $\phi=2 \rho, \phi$ is also independent of $y^{i}$. Transvecting equation (14) by $y^{j}$ and using (6), we get

$$
£ H_{k h}^{i}=\phi H_{k h}^{i}
$$

Differentiating (29) partially with respect to $y^{j}$ and using the fact that $\phi$ is independent of $y^{j}$, we get $£ H_{j k h}^{i}=\phi H_{j k h}^{i}$, which shows that the special concircular $R$-Lie-recurrence is an $H$-Lie-recurrence.

\section{Special Concircular R-Lie-Recurrence in a Birecurrent Finsler Space}

Definition 4. A birecurrent Finsler space $F_{n}$ characterized by

$$
R_{j k h|l| m}^{i}=a_{l m} R_{j k h}^{i},
$$

where $a_{l m}$ are components of a non-zero covariant tensor of type $(0,2)$ and $R_{j k h}^{i} \neq 0$ (see [8] and [10]).

Theorem 5. A birecurrent Finsler space $F_{n}(n>2)$ admitting a special concircular $R$-Lie-recurrence necessarily satisfies the conditions $\phi=3 \rho$ and $v^{r} a_{m r}=\rho_{m}$.

Proof. Suppose that this space admits a special concircular $R$-Lie-recurrence characterized by equations (15) and (14). In view of (11), equation (14) may be written as

$$
R_{j k h \mid r}^{i} v^{r}=(\phi-2 \rho) R_{j k h}^{i}
$$


Differentiating equation (31) covariantly with respect to $x^{m}$, we get

$$
v_{\mid m}^{r} R_{j k h \mid r}^{i}+v^{r} R_{j k h|r| m}^{i}=\left(\phi_{m}-2 \rho_{m}\right) R_{j k h}^{i}+(\phi-2 \rho) R_{j k h \mid m}^{i}
$$

where $\phi_{m}=\phi_{\mid m}$. Using (15a) and equation (30) in equation (32), we have

$$
\left(v^{r} a_{r m}-\phi_{m}+2 \rho_{m}\right) R_{j k h}^{i}=(\phi-3 \rho) R_{j k h \mid m}^{i} .
$$

In view of the definition for a birecurrent Finsler space, $R_{j k h}^{i} \neq 0$. In equation (33), for $R_{j k h \mid m}^{i} \neq 0$, implies $a_{l m}=0$, a contradiction. Therefore, equation (33) implies either of the follwing conditions:

$$
\begin{aligned}
& \text { (i) } \phi-3 \rho=0, \quad v^{r} a_{m r}-\phi_{m}+2 \rho_{m}=0, \\
& \text { (ii) } \phi-3 \rho \neq 0, \quad v^{r} a_{m r}-\phi_{m}+2 \rho_{m} \neq 0 .
\end{aligned}
$$

We can write the condition $(i)$ as $\phi=3 \rho, v^{r} a_{m r}=\rho_{m}$.

Let us consider the condition $(i i)$. In this case equation (33) may be written as

$$
R_{j k h \mid m}^{i}=\frac{\left(v^{r} a_{m r}-\phi_{m}+2 \rho_{m}\right)}{\phi-3 \rho} R_{j k h}^{i},
$$

which shows that the space is $R$-recurrent. In view of theorem 1 , an $R$-recurrent Finsler space $F_{n}(n>2)$ does not admit a special concircular $R$-Lie-recurrence. Therefore, the conditions $(i i)$ is not possible.

\section{Special Concircular $R$-Lie-Recurrence in a Bisymmetric Finsler Space}

Definition 6. A bisymmetric Finsler space $F_{n}$ characterized by [9]

$$
R_{j k h|l| m}^{i}=0
$$

Theorem 7. An R-bisymmetric Finsler space $F_{n}$ admitting a special concircular $R$-Lie-recurrence with condition $\phi=3 \rho$ is flat.

Proof. Suppose that this space admits a special concircular $R$-Lie-recurrence characterized by equations (15) and (14). Differentiating equation (31) covariantly with respect to $x^{m}$, we have

$$
v_{\mid m}^{r} R_{j k h \mid r}^{i}+v^{r} R_{j k h|r| m}^{i}=\left(\phi_{m}-2 \rho_{m}\right) R_{j k h}^{i}+(\phi-2 \rho) R_{j k h \mid m}^{i}
$$


Using equations (15a) and equation (35) in equation (36), we get

$$
(\phi-3 \rho) R_{j k h \mid m}^{i}=\left(2 \rho_{m}-\phi_{m}\right) R_{j k h}^{i} .
$$

If $\phi=3 \rho$, equation (37) reduces to $\rho_{m} R_{j k h}^{i}=0$ which implies $R_{j k h}^{i}=0$ for $\rho_{m} \neq 0$.

Theorem 8. An R-bisymmetric Finsler space $F_{n}(n>2)$ admitting a special concircular $R$-Lie-recurrence with $\phi=2 \rho$ is a $R$-symmetric Finsler space.

Proof. If $\phi=2 \rho$ then $\phi_{m}=2 \rho_{m}$. Therefore, equation (37) may be written as

$$
R_{j k h \mid m}^{i}=0 .
$$

This shows that the space is symmetric. Thus, we see that a $R$-bisymmetric Finsler space admitting a special concircular $R$-Lie-recurrence with $\phi=2 \rho$ is a symmetric space admitting a special concircular $R$-Lie-recurrence.

From Theorem 3 and Theorem 8, we may conclude:

Theorem 9. A special concircular $R$-Lie-recurrence in a non-flat $R$ bisymmetric Finsler space $F_{n}(n>2)$ with $\phi=2 \rho$ is an $H$-Lie-recurrence and the $R$-bisymmetric Finsler space $F_{n}(n>2)$ is necessarily of constant Riemannian curvature.

Theorem 10. An R-bisymmetric Finsler space $F_{n}(n>2)$ can not admit a special concircular $R$-Lie-recurrence if $\phi$ is neither $2 \rho$ nor $3 \rho$.

Proof. If $\phi \neq 2 \rho$ and $\phi \neq 3 \rho$, then equation (37) may be written as

$$
R_{j k h \mid m}^{i}=\frac{2 \rho_{m}-\phi_{m}}{\phi-3 \rho} R_{j k h}^{i} .
$$

This shows that the space is recurrent, but in view of Theorem 1 , a recurrent space does not admit a special concircular $R$-Lie-recurrence.

From Theorems 7, 8, 9 and 10 we may conclude:

Theorem 11. An R-bisymmetric Finsler space $F_{n}(n>2)$ admitting a special concircular $R$-Lie-recurrence is either flat or a Finsler space of constant Riemannian curvature. 


\section{Acknowledgments}

The first author is financially supported by UGC, Government of India.

\section{References}

[1] C.K. Mishra, Gautam Lodhi, On Curvature Inheriting Symmetry in Finsler Space, Acta Univ. Apulensis Math. Inform., 30 (2012), 39-48.

[2] H. Rund, The Differential Geometry of Finsler Spaces, Springer-Verlag, Berlin (1959).

[3] K.L. Duggal, Curvature Inheriting Symmetry in Riemannian Space with Applications of Fluid Space Times, J. Math. Phys., 33, 9 (1992), 2989-2997.

[4] K. Yano, The Theory of Lie-derivative and its Application, North-Holland Publishing Co., Amsterdam (1957).

[5] P. N. Pandey, On Lie recurrent Finsler Manifolds, Indian J. Math., 24, 1-3 (1982), 135143.

[6] P.N. Pandey, A Symmetric Finsler Manifold with a Concircular Vector Field, Proc. Nat. Acad. Sci. India Sect., A54, 3 (1984), 271-273.

[7] P.N. Pandey, A Recurrent Finsler Manifold with a Concircular Vector Field, Acta Math. Acad. Sci. Hungar., 35, 3-4 (1980), 465-466.

[8] P.N. Pandey, Certain Types of Affine Motion in a Finsler Manifold. I., Colloq. Math., 49, 2 (1985), 243-252.

[9] P.N. Pandey, On Bisymmetric Finsler manifolds, Math. Education, 11, 4 (1977), A77A 80.

[10] P.N. Pandey, Reema Verma, Infinitesimal Affine Motion in a Finsler Space Equipped with Cartan's Connection, J. Int. Acad. Phys. Sci., 1, 1-2 (1997), 39-54.

[11] P.N. Pandey, S. Saxena, On Projective N-curvature Inheritance, Acta Math. Hungar., 136, 1-2 (2012), 30-38.

[12] P.N. Pandey, Vaishali Pandey, On $K$-curvature Inheritance in a Finsler Space, J. int. Acad. Phys. Sci., 13, 4 (2009), 395-400.

[13] P.N. Pandey, Vaishali Pandey, On K-Lie-recurrence in a Finsler Space, South Asian J. Math., 3, 6 (2013), 402-410.

[14] S. Saxena, P.N. Pandey, On Lie-recurrence in Finsler Spaces, Differ. Geom. Dyn. Syst., 13 (2011), 201-207. 
